\title{
A new method for detecting free liquid moisture on façade surfaces
}

\author{
Ayman Bishara ${ }^{1, *}$, Helge Kramberger ${ }^{1}$ \\ ${ }^{1}$ Dr. Robert-Murjahn-Institut (RMI), Industrie St. 12, D-64372 Ober-Ramstadt, Germany
}

\begin{abstract}
Liquid water present on the surface of façades is significantly responsible for microorganism growth. This issue became especially important with the increasing use of thermal insulation. The formation of condensation on these façades is often increased due to low surface temperatures [1]. Therefore, the development of a method for controlling the wetness and drying of façades is necessary, since no suitable method is yet available for this purpose. In this paper, the development of an active façade moisture detection system is presented. This system consists of a surface humidity sensor network based on reflection from infrared (IR) radiation, data acquisition and intelligent processing technology. The system is tested and calibrated in the lab for different textured surfaces. Different newly developed coating systems are tested in-situ using this method. These coatings are evaluated with respect to their surface condensation in order to identify the resistance against condensation. The method provides long-term non-destructive measurements of surface wetness. Targeted measures to prevent or reduce the growth of algae can be initiated with the obtained data. In addition, the measured data make a valuable contribution to the development of facade materials and coatings, which have improved drying properties and balance between hydrophilic and hydrophobic surface. As a result, the use of biocides to protect the facade from algae growth can be reduced.
\end{abstract}

\section{Introduction}

Biocide-free and algae growth resisting coating systems have been researched for years. Suitable methods for measuring the free moisture on a façade are still missing. Moreover, only the surface moisture is relevant for the microorganism growth and not the absorbed moisture of the coating system. Therefore, the goal is a method that could detect the free surface moisture, independently of absorbed water.

The actual detection of surface wetness is currently being carried out by renowned research institutes in a manual process [2]. For this purpose, surface moisture is taken off from the facade with an absorbent textile and then the weight increase of the textile is determined. Accordingly, long-term measurements are limited and possible only at easily accessible locations. However, this method is inaccurate and can only determine the amount of surface moisture, but not the duration in which surface moisture is present.

* Corresponding author: ayman.bishara@dr-rmi.de 
Another method is to determine the weight of a model plate, where the moisture is detected also gravimetrically, whereby no differentiation is possible between the absorbed and the surface moisture. Therefore, this method is unsuitable to determine the proportion of surface moisture to the total moisture. Furthermore this method is not applicable to real building and large surfaces. The calculation of the dew water also provides no information about the duration of the surface moisture. Since the duration of surface moistening is more relevant and important than the amount of surface moisture itself, the development of a new measurement method that takes into account the above-mentioned aspects and that provides reasonable and reliable measurement is necessary. In this research context, it was determined during the experimental series in RMI that the intensity of the reflected light depends on the surface moisture. This correlation forms the basis for an optical detection of surface moisture.

\section{Surface wetness detection system (ONE)}

The basic technical principle of the measuring method is the reflection of light on waterwetted surfaces, since the intensity of the reflected infrared (IR) radiation is dependent on the degree of surface wetness (amount of moisture). The following scheme (Figure 1) illustrates the structure of the system including all components and conditions. The real challenges are: environment conditions (brightness, temperature, and humidity), roughness, colour and material of the tested surface, the duration of measurement and the volumes of data as well as the stability of the system.

The system consists of a suitable, physically complex light emitter / sensor. The transmitter is an infrared emitter that emits light in the wavelength range of $900 \mathrm{~nm}$ on the test surface, which makes it possible to separate the measuring signal with optical filters from the daylight. In addition, this high-power IR light is pulsed onto the test area in the watt range. In order to obtain a further possibility of differentiation from strong solar radiation, the sensor electronics are correlated with the usual pulse frequency (about 30 $\mathrm{kHz}$ ). The IR receiver uses a photodiode array (semiconductor device for light measurement, pre-amplification and signal filtering) and is equipped with a daylight blocking filter that only allows wavelengths in the $900 \mathrm{~nm}$ range to pass through.

The sensor is developed as a pin diode array with integrated electronics for preamplification and signal filtering. It has also various filters for daylight compensation.

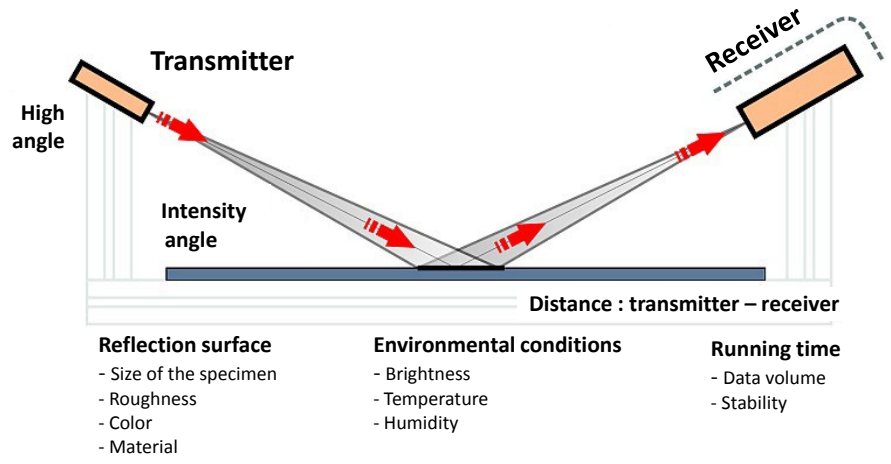

Fig. 1. System principal including components and conditions

The objective of the system is achieved by a method consisting of the following steps: Arrangement of the light source spaced from the surface, so that the beam path hits at an angle of incidence on the surface area. The total reflection of the incident light beam on water which adheres to the surface must be set at a reflection angle, so that the beam path of 
a reflected light is formed. The reflected light beam must be detected by a light sensor, which is arranged in the beam path of the reflected light beam. The output signal of the light sensor must be evaluated; whereby higher surface moisture is assigned to a higher reflectance of the reflected light beam [3].

\subsection{Calibration}

Since the system works on a basic optical principle, the particular challenge of this work is to be able to measure all types of plaster and surface from smooth to rough, with a wide variety of colour and protective coatings under the most adverse environmental conditions with one device and without complex settings on site. However, the plasters differ not only in their grain size, but also in their chemical-physical properties to bind or repel water (see figure 2). All of these factors have a strong effect on the reflection properties of the adherent water and significantly affect the quality of the IR measurement signal. In addition, various facade paints on these plasters have a different absorption capacity with respect to the IR transmission radiation [4].

The preliminary laboratory experiments have proved that the intensity of the reflected IR radiation depends clearly on the degree of surface wetness. In our laboratory setup, the reflection angle has been optimized in order to obtain a significant useful signal for each plaster variant. In order to avoid the environmental influences such as rain or sun at the measuring point by transmitter or receiver, the system should work contactless. The transmitter and receiver related to the experiment must each be at a distance of $30-50 \mathrm{~cm}$ from the actual measuring point. Furthermore, the tests show also that the optimization of the optical channel increases the effect of the measuring significantly. By additional compensation and control algorithms, the measurement result can be also considerably stabilized and largely independent of environmental influences.

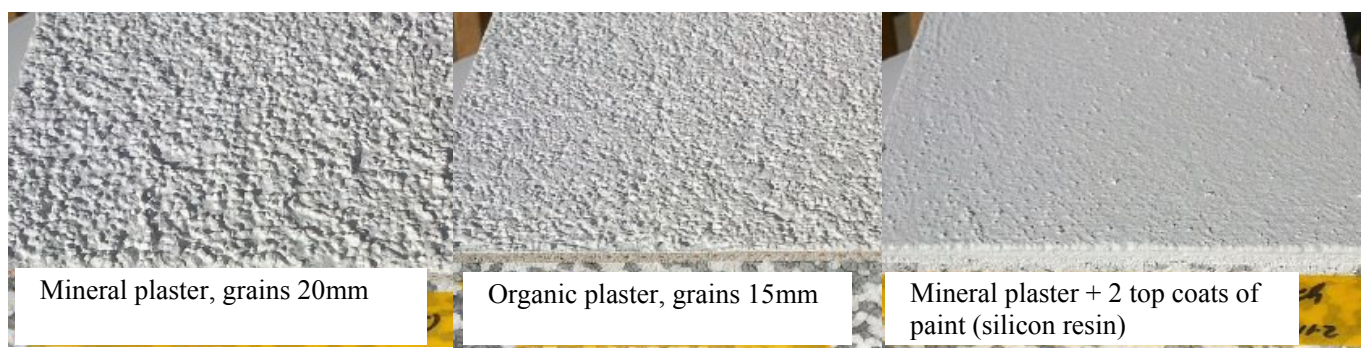

Fig.2. examples of some kind of plasters with different grain size

The measurement system is calibrated based on an optical principle, therefore a reference surface is provided. By a defined dry surface, the ONE-System has to display a $95 \%$ reflectance (value close to the maximum limit " $100 \%$ " to observe the variation and swinging of the refection). This requires a special internal calibration of the system during its manufacturing. First, for this purpose, in addition to the input amplifier and the filter stages of the measuring light sensor, the infrared intensity of the radiation source is adjusted so that a defined useful signal level is available. After that, various calibration procedures are performed for internal analogue to digital converters (AD) with pulse width modulation (PWM) control stages until the system can finally be used for application. The integrated sensor stages for measuring temperature and ambient light must also be adjusted separately. Hence, the absolute reflection at any surface (from smooth to rough) is between 0 and 999 digits regardless of whether the surface is dry or wet). The calibration value for any surface is between 0 to $100 \%(0 \%$ : surface is dry, $100 \%$ the surface is fully wet. Thus, the calibration process for any surface (depending on its roughness, colour, and physical 
and chemical properties of the material) can be easily carried out. If the absolute refection value in dry-case is e.g. 135 and in wet-case 700 that means $135=0 \%$ and $700=100 \%$ (calibrated value).
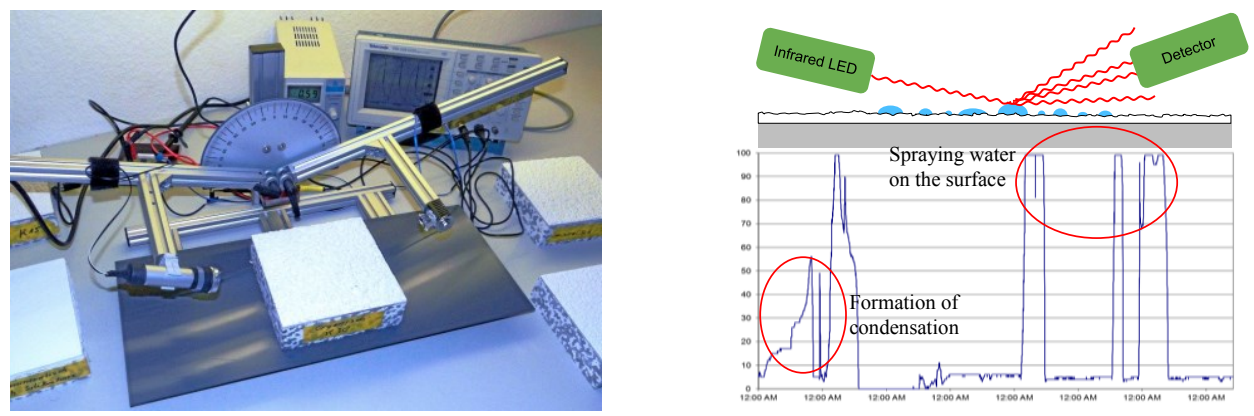

Fig. 3. System calibration, Laboratory setup to optimize the reflection angle and distance transmitterreceiver on different coating systems, right: example for laboratory calibrated results

\section{Outdoor experiment}

With the goal of investigating the functionality of the ONE-System, described above, and of different pigments (conventional and modified pigments) with regards to their resistance to wetness formation on the surface, a case study in middle Germany was created. Four test walls have been established in the test field of Dr. Robert-Murjahn-Institute under real climate conditions. All walls have the same structure: a massive wall insulated with external thermal insulation composite system (ETICS) using polystyrene board (EPS). Each wall is coated with three different pigments, the first one with conventional pigments (BW) and second one with modified pigment (WW). WW-pigments is modified to be more resistant against the formation of condensation on its surface and thus to avoid the algae growth. The four walls are oriented in two different directions as follows: three conventional pigments, one in south direction (S.BW), second one in north direction (N.BW), third modified pigment, one is south oriented (S WW), second one north oriented (N. WW). Figure 4 shows one exemplar of the test walls including the colour formulation label, left with conventional pigments and right with modified pigments. Figure 5 shows two of the test walls, which are oriented into the south direction in addition to the weather station (pyranometer, rain, humidity and temperature sensors) and ONE-measuring systems installed on the test walls.
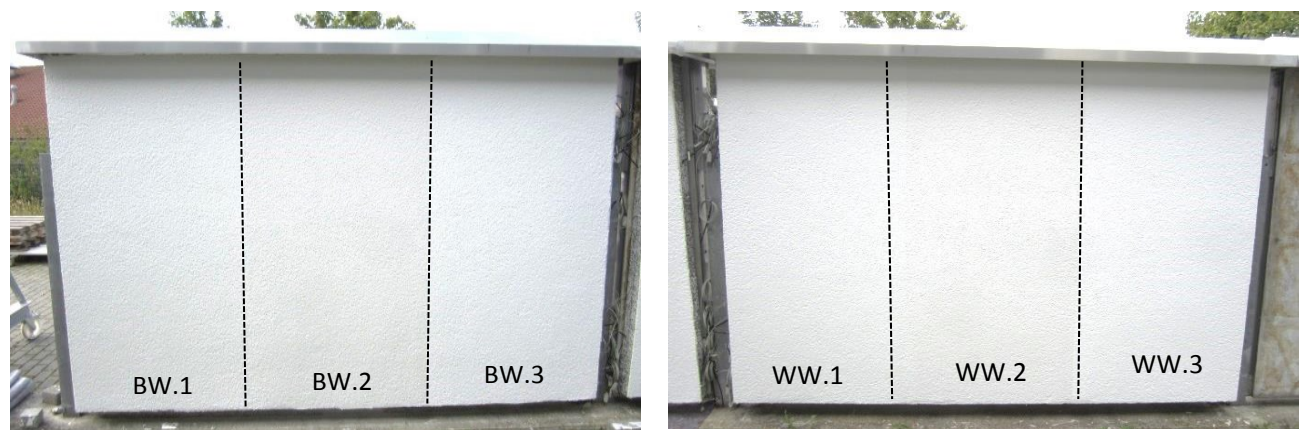

Fig.4. example of the test walls, left: including conventional pigments (BW), right: including modified pigments (WW) 

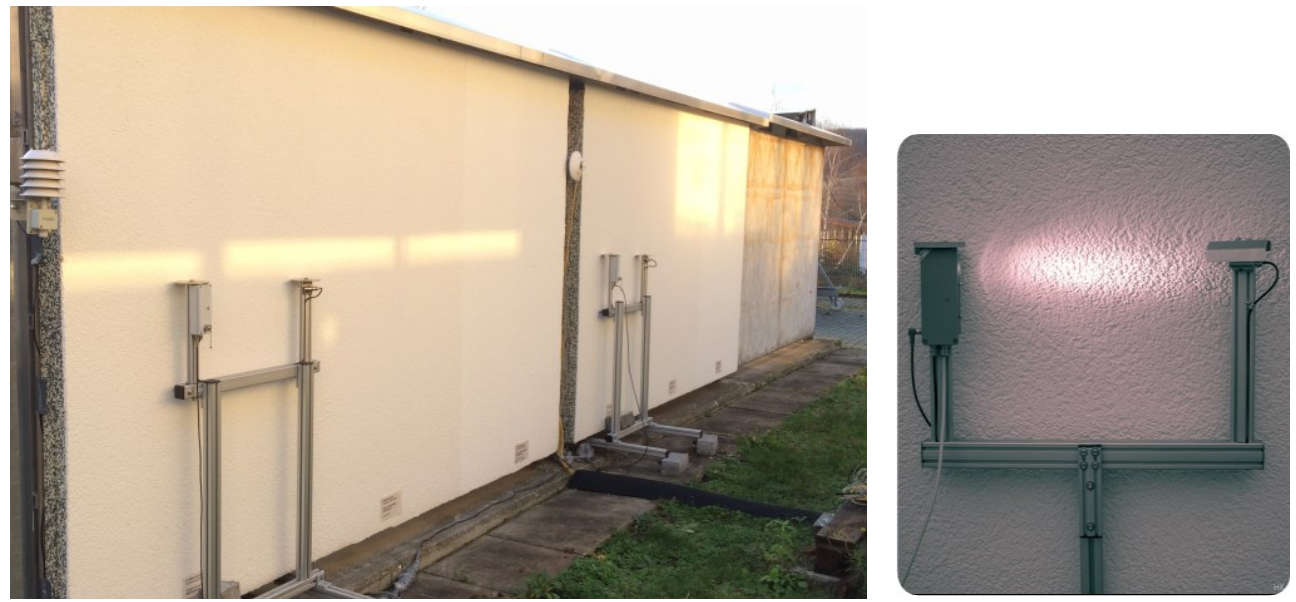

Fig. 5. ONE-System installed on a test wall, right: considered measuring zone (illuminated area)

\subsection{Measurement results and assessment}

In addition to the detection of surface moisture using the ONE-System, the surface temperature, outside air temperature, relative humidity and driving rain are measured. The measurement has been started in February 2018 and is still running.
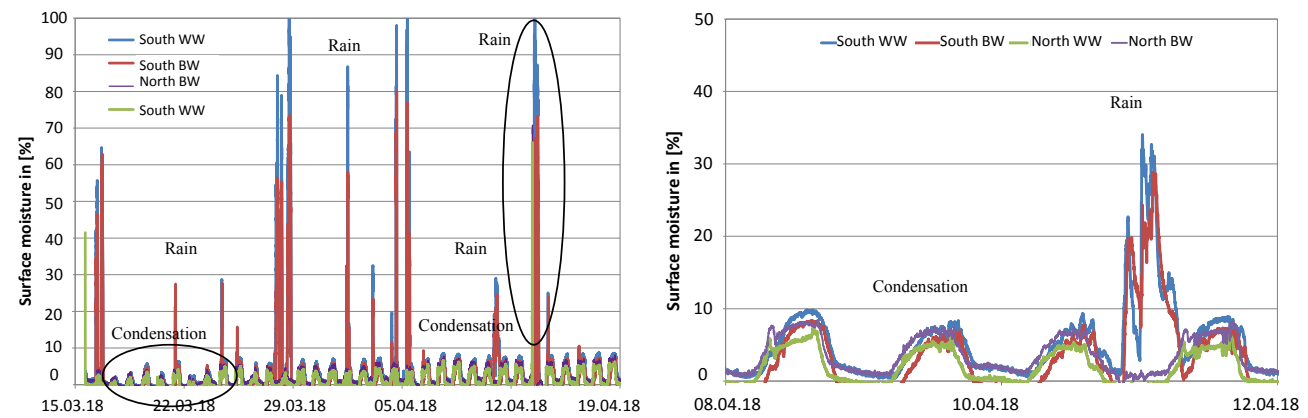

Fig.6. surface moisture measured by ONE-System (March $15^{\text {th }}-$ April $19^{\text {th }}$ ), right: same measurements in 4- day's period (April $8^{\text {th }}-$ April $12^{\text {th }}$ )
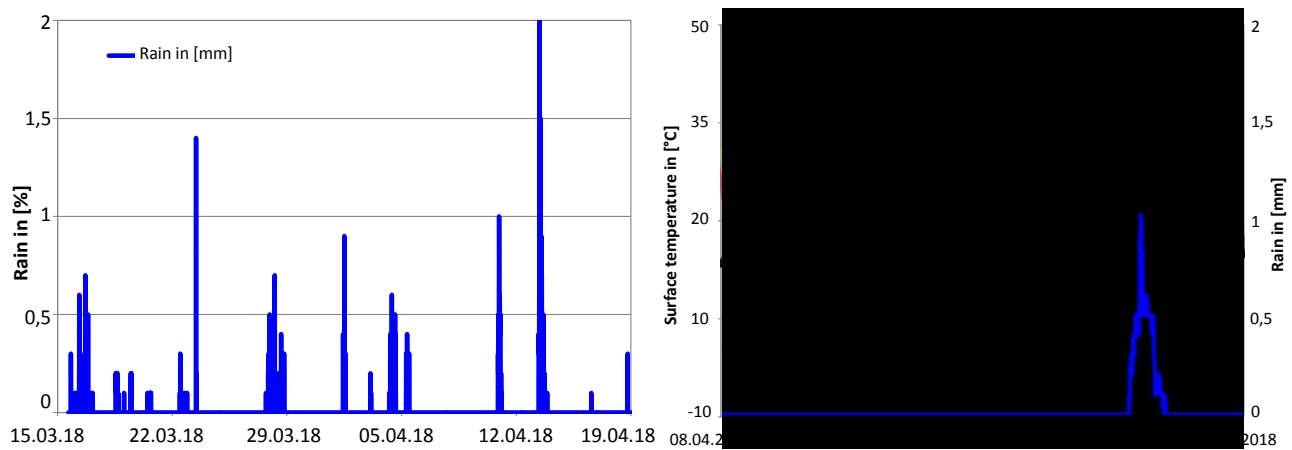

Fig.7. Driving rain in test field (from March $15^{\text {th }}-$ April $19^{\text {th }}$ ), rain, right: surface temperature of all pigments and calculated dew point 
Figure 6 (left) shows the surface moisture measured by ONE-System between March $15^{\text {th }}$ and April 19 $9^{\text {th }}$ 2018; figure 6 (right) presents the same measurements in a 4- day's period in large scale. Driving rain measurement (in $\mathrm{mm}$ ) from March $15^{\text {th }}$ to April $19^{\text {th }}$ 2018 is demonstrated in figure 7 (left). Figure 7 (right) presents in addition to the driving rain (in $\mathrm{mm}$ ) the surface temperature of all pigments (in north and south direction) as well as the calculated dew point in a 4 days period (from April 8 to April 12 2018).

The results of the surface moisture measurement show that condensation and driving rain can be very well differentiated. This can clearly be seen in the comparison of figure 6 and 7 (left). The peaks of surface moisture recorded by the ONE-System (fig 6) correlates very well to the rain peaks in figure 7 . The results of the surface moisture measured by ONE-System correspond also well with the measured surface temperature and the relating calculated dew point. When the surface temperature drops below the dew-point, the ONESystem measures the surface moisture at about $10 \%$. According to the results of both the ONE-System and the surface temperature, it can be observed that modified pigments (WW) have some advantage in comparison to the conventional pigments. The measurement shows that their surface temperature is a little bit higher and thus the formation of condensation on their surface is still smaller than the condensation on the surface of BW-pigments. Moreover, this observation depends on the time (day/night) and on the orientation. WW pigment gives better results by daylight and in north orientation. However, this advantage is only observable in minimum range, thus it needs a further investigation in order to increase its function.

\section{Conclusions}

On the basis of optical and reflection technology, an innovative measurement system for the surface moisture (ONE-System) has been developed. To ensure a long-term and efficient measuring system, daylight filters have been established and tested. Using this advanced technology, non-destructive effective measurements and diagnosis about the current state of surface moisture have been recorded and evaluated.

The results of the case study in Central Germany (4 test walls) the ONE-sensor system shows after more than one year of measuring that the system is robust and works very precisely even under very hard outside conditions (rain, sun radiation, frost etc.). During the observation period, dew is the much more relevant source of moisture at least for the duration of the surface moisture. The measurements of the ONE-System show also very good correspondence between the rain data, the measured surface temperature and the related calculated dew point. This study proves a reliable method for long-term monitoring the surface moisture, which makes a valuable contribution to the development of facade materials and coatings by improving of its drying, hydrophilic und hydrophobic properties.

\section{References}

1. Blaich, J.: External walls with thermal insulation composite system, algae and fungus growth. German architect sheet 31, H. 10 S. 1393-1394 (1991)

2. Kraus, M., Sedlbauer, K.: Instationär Feuchtegehalt an Außenoberfläche und seine Auswirkungen auf Mikroorganismus (2003)

3. Bishara, A., Kramberger, H.: Wireless Sensor System for Intelligent Facades, 7th International Building Physics Conference, IBPC (2018)

4. Bishara, A., Kramberger, H., Ptatschek, V.: Influence of different pigments on the surface temperature of ETICS. Elsevier Ltd (2017) 